\title{
论 文 嫦娥五号轨道测量与确定技术专题
}

\section{基于轨道共面交会的月面上升器起飞参数计算 方法}

李革非 ${ }^{1,2^{*}}$, 刘勇 ${ }^{1,2}$

1. 北京航天飞行控制中心, 北京 100094;

2. 航天飞行动力学技术重点实验室, 北京 100094

*联系人, E-mail: sophiebacc@sina.com

收稿日期: 2021-03-07; 接受日期: 2021-04-30; 网络出版日期: 2021-10-19

装备预研重点实验室基金(编号: 6142210190105)资助项目

摘要 针对上升器与轨道器以轨道共面实现控制量优化的月球轨道交会问题, 提出了精确计算上升器起飞参数 的方法. 根据上升器月面起飞位置和轨道器环月轨道, 利用环月轨道与起飞点共面的球面三角关系, 给出了计算 起飞方位角和起飞时刻初值的解析模型; 基于上升器月面上升特征参数计算了上升器入轨轨道; 采用四脉冲远程 导引控制策略实现上升器交会轨道机动, 建立了远程导引终点轨道平面偏差到入轨点轨道参数偏差的映射关系; 推导了基于轨道倾角偏差和升交点赤经偏差修正起飞方位角和起飞时刻的解析算法; 设计了精确月面起飞参数 的迭代计算流程. 仿真验证了上升器起飞参数初值方法和修正方法的正确性和快速收敛性, 基于轨道共面交会获 得的精确月面起飞参数有效消除了远程导引终点法向偏差，实现了无需轨道平面修正控制量的优化交会轨道控 制, 节省了推进剂消耗. 可应用于从月球和其他地外天体起飞进行交会对接的起飞参数优化确定.

关键词月面起飞, 起飞方位角, 起飞时刻, 交会对接, 远程导引

PACS: 02.60.Gf, 02.60.Pn, 95.55.Pe, 97.75.Pq

\section{1 引言}

2020年11月24日，嫦娥五号探测器发射升空开启 了我国首次地外天体采样返回之旅；2020年12月17日， 嫦娥五号返回器成功着陆标志着我国首次地外天体采 样返回任务圆满成功. 嫦娥五号实现了我国航天史上 首次月面采样、月面起飞、月球轨道交会对接、带样 返回等多个重大突破, 是我国迄今为止系统最复杂、
技术难度最大的航天工程.

叶培建等人 ${ }^{[1]}$ 提出有人参与的深空探测工程是无 人深空探测工程和载人航天工程的有机结合, 着陆地 外天体并安全起飞返回地球是面临的风险和技术挑战 之一。果琳丽等人 ${ }^{[2]}$ 在载人月面着陆及起飞技术初步 研究中, 指出载人登月任务中最具特色的就是载人月 面着陆及起飞过程. 彭兢等人 ${ }^{[3]}$ 提出月面起飞上升过 程应主要关注五个方面：(1) 月面上升过程选择;

引用格式: 李革非, 刘勇. 基于轨道共面交会的月面上升器起飞参数计算方法. 中国科学: 物理学 力学 天文学, 2021, 51: 119502 Li G F, Liu Y. Method for calculation of takeoff parameters of lunar ascent module based on orbital coplanar rendezvous (in Chinese). Sci Sin-Phys Mech Astron, 2021, 51: 119502, doi: 10.1360/SSPMA-2021-0056 
月面上升制导控制律; (3) 起飞上升推力选择; (4) 月面 上升初始基准, 包括上升器起飞姿态、起飞时间、起 飞点位置, 以及入轨点位置和速度目标等; (5) 月面起 飞稳定性.

针对月面起飞过程的自主对准问题，王卫华等 人 $^{[4]}$ 提出了采用星间链路、天文观测以及惯性测量的 组合导航方法, 获得高精度着陆器位置, 为月面起飞提 供可靠性保障. 对于上升器从月面起飞, 经过连续动力 飞行、进入预定轨道的月面上升过程，国内外有较多 文献对月面上升制导控制律进行了研究. 针对月面上 升的轨迹优化问题，通常采用通过间接法转化为两点 边值问题或通过直接法转化为非线性规划问题，以燃 料消耗最少为性能指标求解最优控制 ${ }^{[5-7]}$. Hull等人 ${ }^{[8,9]}$ 采用间接法推导的燃料次优制导律求解了月面上升运 动轨迹. 李金等人 ${ }^{[10]}$ 、马克茂和陈海鹏 ${ }^{[11]}$ 分别采用粒 子群算法和前向扫描法对上升器轨迹优化的两点边值 问题进行了求解. 张磊 ${ }^{[2]}$ 建立以燃料消耗最优为目 标、考虑入轨约束的轨迹优化模型, 通过Gauss伪谱法 和序列二次规划求解上升过程最优推力方向, 开展了 月面上升的轨迹优化与参数分析.

无论是月球采样返回任务还是载人登月任务，月 球轨道交会对接方案通常是首选方案. 嫦娥五号任务 采用了一次发射月球轨道交会对接方案 ${ }^{[3]}$. 典型的载 人月球探测模式设计为，在完成月面任务后，航天员 搭乘载人月面着陆器从月面起飞上升，与在环月轨道 飞行的目标飞行器交会对接 ${ }^{[13-15]}$. 与月面起飞相关联 的飞行控制过程就是月球轨道交会对接. 陈欢等人 ${ }^{[16]}$ 和侯振东等人 ${ }^{[17]}$ 基于共椭圆快速交会策略，给出了任 务周期、推进剂消耗等性能指标最优的环月交会策略 和多约束下载人月面上升交会轨道参数可行域快速求 解方法. 祝海等人 ${ }^{[18]}$ 设计了环月轨道一天快速交会任 务的调相变轨方案，分析了快速交会的调相终端控制 精度、最优初始相位角范围等任务特性参数. 汪中生 等人 ${ }^{[19]}$ 对国内外月球轨道交会远程导引段多脉冲变 轨策略进行了比较分析，在考虑月球轨道交会测控资 源有限和航天器所带燃料受限等特点的基础上, 确定 了我国月球轨道交会远程导引段的 4 脉冲变轨策略方 案. 陈海朋等人 ${ }^{[20]}$ 针对载人登月短期全球访问任务月 面上升过程存在上升舱与返回舱异面交会问题，以减 小调面机动燃料消耗为目标，给出了全月面到达着陆 轨道与上升轨道倾角的求解方法.
综上可知，关于月面上升轨迹控制和月球交会轨 道控制的相关研究较多, 但对于月面起飞参数的确定 和计算的文献较少. 由于月球轨道交会多脉冲变轨优 化使用推进剂是月球探测任务必须考虑的关键问题, 本文通过优化确定与月球轨道交会相关联的月面起飞 参数, 使得上升器起飞上升入轨后, 与轨道器以轨道 共面方式实现交会, 上升器远程导引控制无轨道平面 修正控制量, 从而实现控制量优化的月球轨道交会 控制.

\section{2 坐标系定义和参数说明}

(1) 月面起飞点惯性坐标系 ${ }^{[12]}$ : 在起飞时刻, 原点 $a$ 位于月面起飞点， $x a$ 在月面起飞点当地水平面内指向 飞行方向，其与北向夹角 $A$ 为起飞方位角(东偏为正), $y a$ 沿当地铅垂方向向上, $x a y$ 平面为名义上升平面, 如 图1所示. 图中, $o x_{m} y_{m} z_{m}$ 为月心瞬时主轴惯性坐标系, $\lambda_{a}, \varphi_{a}$ 分别为起飞点经度和纬度.

(2) 月心固连坐标系(主轴坐标系) ${ }^{[12]}$ : 原点为月球 质心, $Z$ 轴方向是月球的自转轴方向, 基本平面为过月 心并与自转轴方向垂直的月球真赤道面， $X$ 轴指向基 本平面与经过月面“中央湾”子午面的交线方向，即月 球赤道面上指向地球方向的惯性主轴方向.

（3）月心瞬时主轴惯性坐标系：在定义时刻(如起 飞时刻)与月心固连坐标系重合. 该坐标系为惯性坐标 系, 用于描述探测器轨道与月球的月理关系.

(4) J2000月心惯性坐标系: 原点位于月心, 以历元 $\mathrm{J} 2000.0$ 地球平赤道、平春分点为参考基准, $X$ 轴平行 于地心天球参考系 $X$ 轴方向; $Z$ 轴平行于地球自转轴,

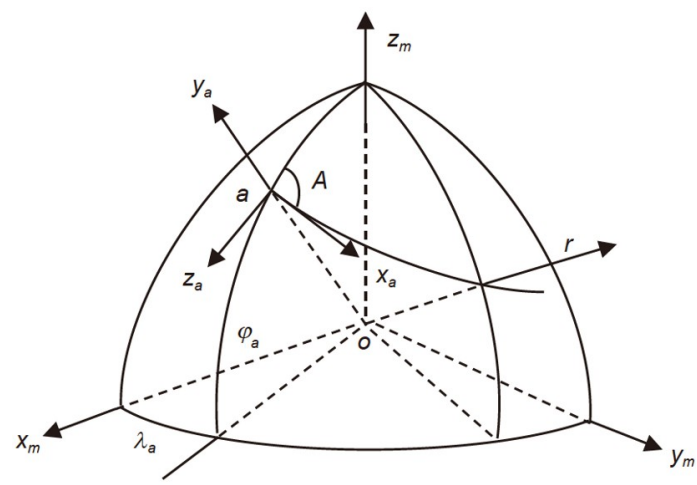

图 1 月面起飞点惯性坐标系

Figure 1 Lunar takeoff site inertial coordinate system. 
指向北极; $Y$ 轴与 $X$ 轴、 $Z$ 轴构成右手系.

（5）J2000月心惯性坐标系与月心瞬时主轴惯性坐 标系的转换：根据历元时刻 $t$ 可计算J2000月心惯性坐 标系到月心固连坐标系的转换矩阵 $\mathbf{M}_{\mathrm{FI}}$. 由于月心瞬 时主轴惯性系在历元时刻与月心固连坐标系重合, 因 此, J2000月心惯性坐标系到月心瞬时主轴惯性系的转 换矩阵等于 $\mathbf{M}_{\mathrm{FI}}$.

（6）起飞点惯性坐标系与月心瞬时主轴惯性坐标 系的转换: 根据起飞方位角 $A$, 计算月心瞬时主轴惯性 坐标系到起飞点惯性坐标系的转换矩阵 $\mathbf{M}_{\mathrm{LF}}$,

$$
\mathbf{M}_{\mathrm{LF}}=\mathbf{R}_{y}(A) \cdot \mathbf{R}_{x}\left(-\frac{\pi}{2}\right) \cdot \mathbf{R}_{y}\left(-\frac{\pi}{2}\right) \cdot \mathbf{R}_{y}\left(-\varphi_{0}\right) \cdot \mathbf{R}_{z}\left(\lambda_{0}\right),
$$

其中, $\mathbf{R}_{x}(\cdot), \mathbf{R}_{y}(\cdot), \mathbf{R}_{z}(\cdot)$ 分别为绕 $x$ 轴、 $y$ 轴、 $z$ 轴旋转指 定角度 $(\cdot)$ 的旋转矩阵, $\lambda_{0}, \varphi_{0}$ 为月面起飞点经度、纬度.

(7) 起飞时刻 $t_{0}$ : 上升器从月面着陆器平台起飞的 时刻.

(8) 入轨时刻 $t_{\mathrm{i}}$ : 上升器从月面起飞后, 经过连续动 力飞行的月面上升阶段进入预定轨道. 月面上升阶段 的结束点为上升器入轨点, 该时刻为入轨时刻 $t_{\mathrm{i}}$, 与起 飞时刻 $t_{0}$ 相差上升阶段的飞行时间 $\Delta t$, 即 $t_{\mathrm{i}}=t_{0}+\Delta t$.

\section{3 月面起飞参数初值和入轨轨道}

\section{1 起飞方位角和入轨时刻初值}

根据轨道器环月飞行轨道和上升器月面起飞点位 置, 计算上升器起飞方位角和入轨时刻的初值. 其方法 是利用球面三角公式, 根据轨道倾角和起飞点纬度计 算上升器起飞方位角, 根据轨道器与上升器的升交点 经度之差计算上升器入轨时刻.

已知, 上升器起飞点位置的月面经度、纬度为 $\lambda_{0}$, $\varphi_{0}$; 轨道器环月飞行轨道的历元时刻为 $t^{\mathrm{g}}, \mathrm{J} 2000$ 月心 惯性坐标系的位置、速度为 $\mathbf{r}_{\mathrm{I}}^{\mathrm{g}}, \mathbf{v}_{\mathrm{I}}^{\mathrm{g}}$.

将轨道器 $\mathrm{J} 2000$ 月心惯性坐标系的位置、速 度 $\mathbf{r}_{\mathrm{I}}^{\mathrm{g}}, \mathbf{v}_{\mathrm{I}}^{\mathrm{g}}$ 转换为月心瞬时主轴惯性坐标系的位置、 速度 $\mathbf{r}_{\mathrm{F}}^{\mathrm{g}}, \mathbf{v}_{\mathrm{F}}^{\mathrm{g}}$ :

$$
\mathbf{r}_{\mathrm{F}}^{\mathrm{g}}=\mathbf{M}_{\mathrm{FI}} \mathbf{r}_{\mathrm{I}}^{\mathrm{g}}, \mathbf{v}_{\mathrm{F}}^{\mathrm{g}}=\mathbf{M}_{\mathrm{FI}} \mathbf{v}_{\mathrm{I}}^{\mathrm{g}} \text {. }
$$

由轨道器月心瞬时主轴惯性坐标系的位置、速 度 $\mathbf{r}_{\mathrm{F}}^{\mathrm{g}}, \mathbf{v}_{\mathrm{F}}^{\mathrm{g}}$ 计算月心瞬时主轴惯性坐标系的轨道根数, 得 到轨道器在月心瞬时主轴惯性坐标系的轨道倾角 $i^{\mathrm{g}}$ 和
升交点赤经 $\Omega^{\mathrm{g}}$.

当上升器入轨轨道与轨道器飞行轨道共面时, 两 者轨道倾角一致, 即 $i^{\mathrm{s}}=i^{\mathrm{g}}$.

根据上升器起飞点纬度 $\varphi_{0}$ 和轨道倾角 $i$, 由球面直 角三角公式，计算起飞点在月球赤道上距升交点的经 度差:

$\Delta \lambda=\arcsin \left(\frac{\tan \varphi_{0}}{\tan i^{\mathrm{s}}}\right)$.

当上升器起飞点纬度位于月球北半球时, 对于上 升器升轨入轨模式, 其月心瞬时主轴惯性坐标系的 升交点经度为 $\Omega_{\mathrm{G}}^{\mathrm{s}}=\lambda_{0}-\Delta \lambda$; 对于上升器降轨入轨模式, 其月心瞬时主轴惯性坐标系的升交点经度为 $\Omega_{\mathrm{G}}^{\mathrm{s}}=\lambda_{0}{ }^{+}$ $\Delta \lambda-\pi$.

当上升器起飞点纬度位于月球南半球时, 对于上 升器升轨入轨模式, 其月心瞬时主轴惯性坐标系的升 交点经度为 $\Omega_{\mathrm{G}}^{\mathrm{s}}=\lambda_{0}+\Delta \lambda$; 对于上升器降轨入轨模式, 其月心瞬时主轴惯性坐标系的升交点经度为 $\Omega_{\mathrm{G}}^{\mathrm{s}}=\lambda_{0}-$ $\Delta \lambda-\pi$.

设上升器与轨道器在月心瞬时主轴惯性坐标系的 升交点经度差表示为 $\Delta \Omega_{\mathrm{G}}=\Omega_{\mathrm{G}}^{\mathrm{s}}-\Omega_{\mathrm{G}}^{\mathrm{g}}$.

在 $t^{\mathrm{g}}$ 时刻, 当 $\Delta \Omega_{\mathrm{G}}=\Omega_{\mathrm{G}}^{\mathrm{s}}-\Omega_{\mathrm{G}}^{\mathrm{g}}=0$ 时, 轨道器飞行轨 道经过上升器的月面起飞点, 即上升器在 $t^{\mathrm{g}}$ 时刻入轨 时, 上升器入轨轨道与轨道器飞行轨道共面. 当 $\Delta \Omega_{\mathrm{G}}=\Omega_{\mathrm{G}}^{\mathrm{s}}-\Omega_{\mathrm{G}}^{\mathrm{g}} \neq 0$ 时, 轨道器需要飞行一段时间, 通 过月球自转消除 $\Delta \Omega_{\mathrm{G}}$, 使得在 $t^{\mathrm{s}}$ 时刻轨道器飞行轨道经 过上升器的月面起飞点, 即上升器在 $t^{\mathrm{s}}$ 时刻入轨时, 上 升器入轨轨道与轨道器飞行轨道共面. 因此, 为保证上 升器与轨道器共面, 轨道器飞行时间为

$\Delta t=t^{\mathrm{s}}-t^{\mathrm{g}}=\frac{\Delta \Omega_{\mathrm{G}}}{\omega_{\mathrm{m}}}$,

式中, $\omega_{\mathrm{m}}$ 为月球自转角速度.

因此, 上升器的入轨时刻为

$t^{\mathrm{s}}=t^{\mathrm{g}}+\Delta t$.

上升器在起飞上升过程中保持上升弹道的射向纵 平面基本不变, 即上升器的起飞方位角不变, 根据球面 直角三角公式, 由轨道倾角和起飞点纬度得到起飞方 位角 $A$ :

对于上升器升轨入轨模式, 
$A=\arcsin \left(\frac{\cos i^{\mathrm{s}}}{\cos \varphi_{0}}\right)$.

对于上升器降轨入轨模式,

$A=\pi-\arcsin \left(\frac{\cos i^{\mathrm{s}}}{\cos \varphi_{0}}\right)$.

上述计算的上升器入轨时刻(起飞时刻)和起飞方 位角作为上升器起飞参数初值.

\section{2 上升器入轨轨道}

根据上升器起飞时刻、起飞方位角以及上升器入 轨点特征参数, 计算上升器入轨轨道. 月面上升平面运 动如图2所示. 已知上升器的入轨点为近月点, 其入轨 点特征参数为: 上升段飞行时间 $\Delta t$, 飞行航程 $d$, 近月点 高度 $h_{\mathrm{p}}$, 入轨点飞行速度 $v$, 可得上升器入轨点在起飞 点惯性坐标系的位置、速度为

$\mathbf{r}_{\mathrm{L}}=\left[\begin{array}{c}r \cdot \sin \beta \\ r \cdot \cos \beta-R_{\mathrm{m}} \\ 0\end{array}\right], \quad \mathbf{v}_{\mathrm{L}}=\left[\begin{array}{c}v \cdot \cos \beta \\ -v \cdot \sin \beta \\ 0\end{array}\right]$,

式中, $r=h_{\mathrm{p}}+R_{\mathrm{m}}, \beta=d / R_{\mathrm{m}}, R_{\mathrm{m}}$ 为月球平均半径.

将上升器入轨点在起飞点惯性坐标系的位置、速 度转换为月心瞬时主轴惯性坐标系的位置、速度:

$\mathbf{r}_{\mathrm{F}}=\mathbf{r}_{0}+\mathbf{M}_{\mathrm{LF}}^{\mathrm{T}} \cdot \mathbf{r}_{\mathrm{L}}, \quad \mathbf{v}_{\mathrm{F}}=\mathbf{M}_{\mathrm{LF}}^{\mathrm{T}} \cdot \mathbf{v}_{\mathrm{L}}$,

式中， $\mathbf{r}_{0}$ 为起飞点在月心瞬时主轴惯性坐标系的位 置, $\mathbf{r}_{0}=\left[R_{\mathrm{m}} \cos \varphi_{0} \cos \lambda_{0}, R_{\mathrm{m}} \cos \varphi_{0} \sin \lambda_{0}, R_{\mathrm{m}} \sin \varphi\right]^{\mathrm{T}}$.

根据上升器入轨时刻 $t^{\mathrm{s}}$ 计算 $\mathrm{J} 2000$ 月心惯性坐标系 到月心瞬时主轴惯性坐标系的转换矩阵 $\mathbf{M}_{\mathrm{F}}$, 由上升器 月心瞬时主轴惯性坐标系的位置、速度计算J2000月

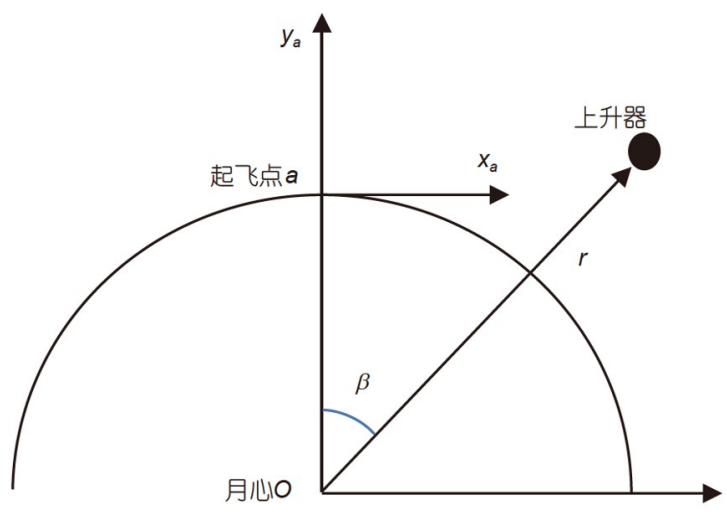

图 2 月面上升的平面运动

Figure 2 Lunar ascending planar movement.
心惯性坐标系的位置、速度:

$\mathbf{r}_{\mathrm{I}}=\mathbf{M}_{\mathrm{IF}} \cdot \mathbf{r}_{\mathrm{F}}, \quad \mathbf{v}_{\mathrm{I}}=\mathbf{M}_{\mathrm{IF}} \cdot \mathbf{v}_{\mathrm{F}} \cdot$

由上升器J2000月心惯性坐标系的位置、速度计 算得到上升器入轨轨道参数.

\section{4 月球交会远程导引控制}

根据上升器入轨轨道和轨道器飞行轨道, 进行上 升器月球交会远程导引轨道控制.

\section{1 轨道器交会对接轨道}

轨道器的交会对接轨道定义为: 根据月球交会对 接任务要求确定某时刻, 在该时刻轨道器环月飞行轨 道为满足指定高度、指定位置要求的圆轨道，同时该 时刻为上升器完成远程导引控制实现与轨道器交会的 终点时刻, 称为远程导引终点 ${ }^{[21]}$.

通常，环月飞行的轨道器经过若干次轨道控制到 达交会对接轨道. 本文中轨道器轨道即为已到达交会 对接轨道.

\section{2 上升器远程导引轨道控制目标}

轨道器在环月交会对接轨道飞行，等待与上升器 交会对接. 上升器从月面起飞上升后进入近、远月点 高度均比轨道器近、远月点高度低的环月轨道，通过 远程导引轨道控制, 实现与轨道器交会. 上升器远程 导引控制的目的是通过控制轨道高度差，缩短相对距 离, 同时消除两航天器轨道平面偏差, 实现上升器与轨道 器的相对位置和相对速度满足远程导引终点要求, 为 上升器和轨道器转入自主控制实现对接创造条件 ${ }^{[21]}$.

上升器远程导引轨道控制是以远程导引终点时刻 两航天器的相对位置和相对速度的状态要求为目标: 在远程导引终点时刻, 轨道器位于一定轨道高度的圆 轨道，上升器进入轨道器前上方或后下方、同一轨道 平面的圆轨道, 与轨道器相距一定距离, 在轨道器轨 道坐标系的相对位置和相对速度表示为 $\Delta x, \Delta y, \Delta z$, $\Delta v_{x}, \Delta v_{y}, \Delta v_{z}, x$ 指向轨道飞行迹向方向, $y$ 指向轨道平 面负法向方向, $z$ 指向月心径向方向, 其中 $\Delta x, \Delta z, \Delta v_{x}$, $\Delta v_{z}$ 是根据两航天器的目标轨道高度差和目标相对距 离的要求确定的目标参数, 法向目标参数要求为 $\Delta y=0$, $\Delta v_{y}=0$. 
远程导引终点相对位置和相对速度表征了上升器 与轨道器在远程导引终点的轨道参数的差异. 迹向和 径向的相对位置和相对速度表征两航天器轨道平面内 参数 $a, e, \omega, f$ 之间的差异, 法向的相对位置和相对速度 表征两航天器轨道平面参数 $i, \Omega$ 之间的差异. 远程导引 终点要求两航天器轨道平面参数 $i, \Omega$ 一致.

\section{3 上升器远程导引轨道控制策略}

本文月球轨道交会远程导引采用经典的4脉冲特 征点变轨交会轨道控制策略 ${ }^{[19-21]}$, 分为轨道平面内控 制和修正轨道面偏差控制，利用轨道面内与轨道面外 参数的解耦特性, 将剥离的轨道平面偏差通过选择起 飞参数予以消除. 具体如下.

(1) 抬高近月点轨道控制. 在远月点执行. 根据轨 道器和上升器相位差和交会总时间, 确定交会转移轨 道的高度. 通过抬高近月点高度达到调整相位的目的, 满足远程导引终点轨道相对迹向位置要求.

(2) 抬高远月点轨道控制. 在近月点执行. 根据两 航天器轨道高度差的要求, 抬高远月点高度; 同时修正 相位调整量. 通过调整远月点高度达到调整轨道半长 轴的目的, 满足远程导引终点轨道相对径向位置要求, 并控制相对径向、切向速度.

(3) 圆化轨道控制. 在远月点附近执行. 按照远程 导引终点轨道高度要求, 圆化上升器轨道, 同时修正相 位调整量. 通过抬高近月点高度达到调整轨道偏心率 尽量为零的要求，满足远程导引终点轨道相对径向位 置和相对切向、径向速度要求.

(4) 轨道平面修正控制. 与三次轨道面内控制解 耦, 在上升器与轨道器的轨道平面的交点执行. 通过 一次组合轨道控制进行轨道倾角和升交点赤经两个偏 差要素的联合修正，满足远程导引终点轨道相对法向 位置和法向速度要求.

上升器远程导引4脉冲轨道控制策略如表1所示, 第一脉冲为抬高近月点轨道控制; 第二脉冲为轨道平 面修正控制；第三脉冲为抬高远月点高度控制；第四 脉冲为圆化轨道控制.

\section{5 月面起飞参数精确计算}

\section{1 起飞参数修正}

在上升器远程导引控制中, 当上升器与轨道器的
表 1 上升器远程导引轨道控制策略 ${ }^{\mathrm{a}}$

Table 1 Ascent module long-range guidance orbit maneuver strategy ${ }^{\text {a) }}$

\begin{tabular}{ccccc}
\hline 序号 & 圈次 & 变轨点 & 变轨方向 & 变轨目的 \\
\hline 1 & $P_{1}$ 圈 & 远月点 & 切向 & 抬高近月点 \\
2 & $N$ 圈 & 轨道平面交点 & 法向 & 修正轨道平面 \\
3 & $P_{2}$ 圈 & 近月点 & 切向 & 抬高远月点 \\
4 & $P_{3}$ 圈 & 远月点附近 & 切向 & 轨道圆化 \\
\hline
\end{tabular}

a) $P_{1}, P_{2}$ 和 $P_{3}$ 分别表示轨道平面内 3 个控制脉冲的轨道圈次, $N$ 表示轨道平面修正脉冲的轨道圈次.

轨道平面存在差异、不共面时，轨道平面修正的控制 量将带来较大的推进剂消耗. 通过对上升器起飞时刻 (入轨时刻)和起飞方位角进行调整，实现上升器入轨 轨道与轨道器飞行轨道共面, 从而达到减少轨道平面 修正控制量、节省推进剂消耗的优化目标.

由于轨道平面修正控制与轨道面内控制是解耦 的, 在远程导引控制时, 只进行轨道平面内三次轨道控 制, 而不进行轨道平面修正控制, 则远程导引终点将会 遗留两航天器法向相对位置和相对速度的偏差, 即轨 道倾角和升交点赤经的偏差.

通过调整起飞方位角修正轨道倾角偏差、调整起 飞方位角和起飞时刻(入轨时刻)修正升交点赤经偏差, 使得无需进行轨道平面修正控制, 即可实现在远程导 引终点两航天器的轨道倾角和升交点赤经一致, 两者 的法向相对位置和相对速度为零.

虽然远程导引终点不经过上升器月面起飞点, 但 远程导引终点两航天器的轨道偏差近似等价于入轨点 两航天器的轨道偏差, 因此, 采用将远程导引终点轨道 平面偏差映射到入轨点轨道平面偏差的思路, 根据远 程导引终点两航天器的轨道倾角偏差和升交点赤经偏 差计算上升器起飞参数的修正量.

设 $i^{\mathrm{s}}$ 和 $\Omega^{\mathrm{s}}$ 分别为上升器在远程导引终点的轨道倾 角和升交点赤经, $i^{\mathrm{g}}$ 和 $\Omega^{\mathrm{g}}$ 分别为轨道器在远程导引终 点的轨道倾角和升交点赤经.

轨道倾角偏差造成的起飞方位角偏差为

$\Delta A=\arcsin \left(\cos i^{\mathrm{s}} / \cos \varphi_{0}\right)-\arcsin \left(\cos i^{\mathrm{g}} / \cos \varphi_{0}\right)$.

轨道倾角偏差和升交点赤经偏差造成的升交点经 度偏差为

$\Delta \Omega_{\mathrm{G}}=\Omega^{\mathrm{g}}-\Delta \lambda^{\mathrm{g}}-\left(\Omega^{\mathrm{s}}-\Delta \lambda^{\mathrm{s}}\right)$, 
式中, $\Delta \lambda^{\mathrm{g}}=\arcsin \left(\frac{\tan \varphi_{0}}{\tan i^{\mathrm{g}}}\right), \Delta \lambda^{\mathrm{s}}=\arcsin \left(\frac{\tan \varphi_{0}}{\tan i^{\mathrm{s}}}\right)$.

通过月球自转消除 $\Delta \Omega_{\mathrm{G}}$ 得到入轨时刻修正量:

$\Delta t=\frac{\Delta \Omega_{\mathrm{G}}}{\omega_{\mathrm{m}}}$

上升器入轨时刻修正为

$t_{\mathrm{i}}^{\mathrm{s}}=t_{\mathrm{i}}^{\mathrm{s}}+\Delta t$.

上升器起飞方位角修正为

$A^{\prime}=A+\Delta A$.

\section{2 起飞参数迭代}

基于轨道共面交会的上升器精确起飞参数需通过 迭代计算获得, 流程如下:

(1) 根据轨道器环月飞行轨道和上升器月面起飞 点位置, 计算上升器入轨时刻(起飞时刻)和起飞方位 角的初值;

(2) 根据上升器起飞时刻、起飞方位角以及上升 器入轨点特征点参数, 计算上升器入轨轨道;

(3) 根据上升器入轨轨道和轨道器飞行轨道, 进行 上升器远程导引控制计算, 只包括轨道平面内的三次 控制，无轨道平面修正控制，获得远程导引终点两航 天器轨道倾角偏差和升交点赤经偏差;

(4) 根据远程导引终点两航天器轨道倾角和升交 点赤经偏差修正上升器起飞方位角和入轨时刻(起飞 时刻);

(5) 使用更新的起飞方位角和入轨时刻(起飞时 刻)，重复步骤(2)-(4)，迭代计算直到远程导引终点升 交点经度偏差 $\Delta \Omega_{\mathrm{G}}$ 小于指定收玫门限 $\varepsilon \Omega_{\mathrm{G}}$;

(6) 最终得到满足轨道共面交会、轨道平面修正 控制量为零的上升器起飞时刻和起飞方位角的优化起 飞参数.

\section{6 仿真校验}

设轨道器环月飞行轨道为高度 $200 \mathrm{~km}$ 的圆轨道, 轨道历元为2020-12-03T11:30:00, 轨道根数为 $a=$ $1937400 \mathrm{~m}, e=0.003, i=29.50^{\circ}, \Omega=110.0^{\circ}, \omega=270.0^{\circ}$, $M=218.0^{\circ}$, 远程导引终点时刻为2020-12-06T02:15:00.

上升器起飞点的月面经度、纬度分别为 $\lambda_{0}=$ $-52.0^{\circ}, \varphi_{0}=43.0^{\circ}$. 上升器采用降轨入轨模式, 入轨点特
征参数为: 飞行时间 $\Delta t=360 \mathrm{~s}$, 飞行航程 $d=250 \mathrm{~km}$, 近 月点高度 $h_{\mathrm{p}}=15 \mathrm{~km}$, 远月点高度 $h_{\mathrm{a}}=180 \mathrm{~km}$, 对应飞行 速度 $v=1709.8405 \mathrm{~m} / \mathrm{s}$.

轨道器与上升器标称月球交会对接轨道分别设计 为 200 和 $210 \mathrm{~km}$ 的环月圆轨道. 月球交会远程导引终点 要求为: 上升器进入轨道器前上方同一轨道平面的圆 轨道, 与轨道器相对距离为 $50 \mathrm{~km}$; 在轨道器轨道坐标 系的相对位置和相对速度为: $\Delta x=50250 \mathrm{~m}, \Delta y=0 \mathrm{~m}$, $\Delta z=-9350 \mathrm{~m}, \Delta v_{x}=12.30 \mathrm{~m} / \mathrm{s}, \Delta v_{y}=0 \mathrm{~m} / \mathrm{s}, \Delta v_{z}=0.32 \mathrm{~m} / \mathrm{s}$.

考虑上升器轨道测定和轨道控制参数上行注入的 测控圈次需间隔4-6圈的需求, 上升器月球交会4脉冲 远程导引控制策略的圈次安排如下.

(1) 第 1 次机动在 4 圈远月点, 抬高近月点.

(2) 第 2 次机动在 9 圈两轨道平面交点, 修正轨道 平面.

(3) 第3次机动在 15 圈近月点, 抬高远月点.

(4) 第4次机动在 21 圈远月点附近, 轨道圆化.

(5) 远程导引终点在 25 圈.

轨道器和上升器轨道动力学模型为月球中心引 力、月球非球形引力摄动 $100 \times 100$ 阶次、地球中心引 力、太阳中心引力以及太阳辐射压摄动.

轨道器质量为 $2000 \mathrm{~kg}$, 面积为 $18 \mathrm{~m}^{2}$, 上升器质量 为 $300 \mathrm{~kg}$, 面积为 $10 \mathrm{~m}^{2}$, 光压系数为 1.4 . 设定起飞参数 迭代计算的远程导引终点升交点经度偏差收敛门限为 $\varepsilon \Omega_{\mathrm{G}}=0.0001^{\circ}$.

起飞参数迭代计算结果见图 3 .

结果表明:

(1) 采用月面起飞初值方法计算的上升器起飞方位 角 $A=99.5968^{\circ}$, 起飞时刻 $\mathrm{T} 0=2020-12-03 \mathrm{~T} 19: 38: 41.96$, 上升器按照无轨道平面修正控制的远程导引控制策 略, 得到远程导引终点法向相对位置和相对速度偏差 分别为 $\Delta y=-520.499 \mathrm{~m}, \Delta v_{y}=3.704 \mathrm{~m} / \mathrm{s}$, 对应的远程导 引终点两航天器轨道倾角偏差 $\mathrm{d} i=-0.053362^{\circ}$, 升交点 赤经偏差 $\mathrm{d} \Omega=0.177938^{\circ}$. 若上升器通过轨道平面修正 控制消除上述偏差, 控制量需 $5.111 \mathrm{~m} / \mathrm{s}$.

(2) 上升器起飞参数经过 11 次迭代计算, 以融合起 飞方位角偏差和起飞时刻偏差的升交点经度合成偏差 作为迭代收玫判断参数，有效简化了对起飞方位角和 起飞时刻的双重判断, 最终得到轨道共面交会的上升 器精确起飞方位角 $A=100.0509^{\circ}$, 起飞时刻 $\mathrm{T} 0=2020$ 12-03T20:30:29.72. 

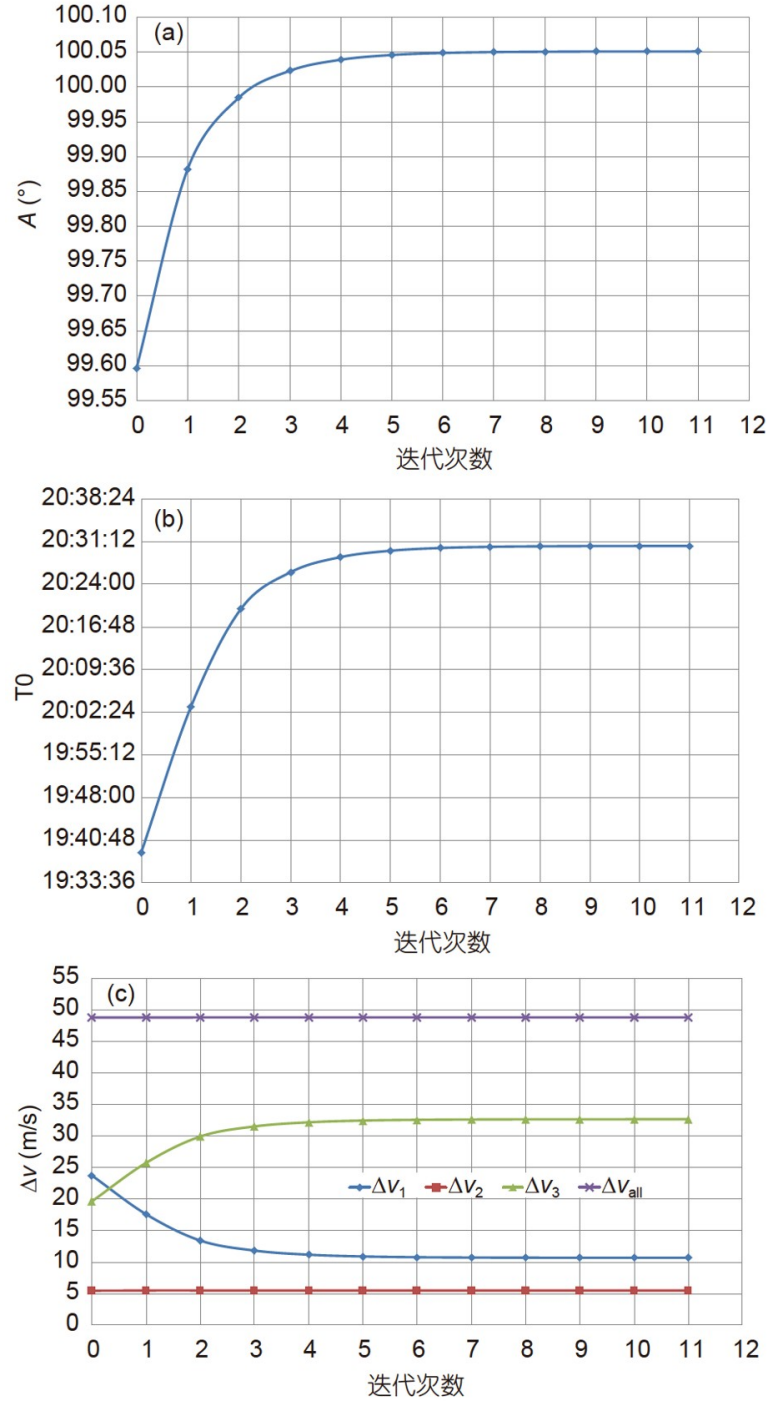

图 3 (网络版彩图)起飞方位角(a)、起飞时刻(b)和脉冲速 度增量(c)迭代计算

Figure 3 (Color online) Iteration of takeoff azimuth (a), takeoff time (b), and three pulses $\Delta v$ (c).

（3）迭代计算中，起飞方位角和起飞时刻快速收 敛. 第4次迭代计算, 远程导引终点法向位置偏差已经 小于 $100 \mathrm{~m}$, 与最终第 11 次迭代结果相比较, 起飞方位 角偏差为 $0.012^{\circ}$, 起飞时刻偏差为 $113 \mathrm{~s}$. 月面起飞参数 偏差对远程导引终点法向偏差的影响具有弱敏感性, 这与月球自转角速度较慢有关. 对于远程导引终点法 向位置偏差小于 $100 \mathrm{~m}$ 的要求，起飞方位角精度达到 $0.01^{\circ}$ 、起飞时刻精度达到 $100 \mathrm{~s}$ 即可满足. 反之，若消 除一定的轨道平面偏差，相应的起飞方位角和起飞时 刻的修正量也较大. 算例中, 为消除远程导引终点轨
道倾角偏差 $-0.053^{\circ}$ 、升交点赤经偏差 $0.178^{\circ}$, 起飞方 位角共修正 $0.4541^{\circ}$, 起飞时刻共修正 $3107.76 \mathrm{~s}$.

(4) 迭代计算中, 远程导引策略的各次控制量分配 有所不同, 以调相功能为主的脉冲 $\Delta v_{1}$ 和 $\Delta v_{3}$ 随迭代计 算逐渐收玫至 10.676 和 $32.644 \mathrm{~m} / \mathrm{s}$, 修正远月点高度的 脉冲 $\Delta v_{2}$ 基本不变 $(5.475 \mathrm{~m} / \mathrm{s})$, 但总的控制量保持不变, 在 $48.782-48.792 \mathrm{~m} / \mathrm{s}$ 之间. 这是由于不同起飞参数对 应的上升器入轨轨道不同, 其相对于轨道器的相位不 同，因此使得上升器轨道面内的调相脉冲相应变化调 整; 但不同入轨轨道的相位均在上升器调相能力范围 内, 因此, 总的控制量保持一致. 算例中, 初值和前2次 迭代的各次控制量变化较大，后续迭代的各次控制量 趋于一致, 表明随着起飞参数的收玫, 上升器入轨轨 道变化较小, 远程导引各次控制量基本不变.

由于上升器起飞参数与轨道器轨道、起飞点月面 位置、上升器远导交会控制策略三者密切相关. 下面 针对起飞点月面位置和轨道器轨道的变化, 进一步分 析验证本文方法的适用性.

(1) 改变起飞点位置

分别考虑起飞点经度变化 $\pm 1^{\circ}$ 、纬度变化 $\pm 0.3^{\circ}$ 的 4种工况. 各工况结果见表 2.

上述结果表明, 受月球由西向东自转的影响, 起飞 点经度向西变化 $1^{\circ}$, 起飞时刻推后约 $2.37 \mathrm{~h}$, 起飞方位 角增大约 $0.235^{\circ}$; 起飞点经度向东变化 $1^{\circ}$, 起飞时刻提 前约 $2.3 \mathrm{~h}$, 起飞方位角减小约 $0.213^{\circ}$. 起飞点纬度向南 变化 $0.3^{\circ}$, 起飞时刻推后约 $4.2 \mathrm{~h}$, 起飞方位角增大约 $1.686^{\circ}$; 起飞点纬度向北变化 $0.3^{\circ}$, 起飞时刻提前约 $6.07 \mathrm{~h}$ ，起飞方位角减小 $2.351^{\circ}$. 起飞点纬度对起飞参 数的影响比经度要大得多.

(2) 改变轨道器轨道参数

分别考虑半长轴增大 $100 \mathrm{~km}$ 、偏心率增大为 0.01 、 轨道倾角增大 $0.5^{\circ}$ 的 3 种工况. 各工况结果如表 3 所示.

上述结果表明, 偏心率变化对上升器起飞参数影

表 2 起飞点位置对起飞参数的影响

Table 2 Takeoff parameters variation with takeoff positions

\begin{tabular}{ccc}
\hline 工况 & 起飞时刻 & 起飞方位角 $\left(^{\circ}\right)$ \\
\hline$\lambda_{0}, \varphi_{0}$ & $2020-12-03 \mathrm{~T} 20: 30: 27$ & 100.051 \\
$\lambda_{0}-1^{\circ}$ & $2020-12-03 \mathrm{~T} 22: 52: 48$ & 100.286 \\
$\lambda_{0}+1^{\circ}$ & $2020-12-03 \mathrm{~T} 18: 12: 07$ & 99.838 \\
$\varphi_{0}-0.3^{\circ}$ & $2020-12-04 \mathrm{~T} 00: 42: 30$ & 101.737 \\
$\varphi_{0}+0.3^{\circ}$ & $2020-12-03 \mathrm{~T} 14: 26: 06$ & 97.700 \\
\hline
\end{tabular}


表 3 轨道参数对起飞参数的影响

Table 3 Takeoff parameters variation with orbit parameters

\begin{tabular}{ccc}
\hline 工况 & 起飞时刻 & 起飞方位角 $\left({ }^{\circ}\right)$ \\
\hline 原轨道 & $2020-12-03 \mathrm{~T} 20: 30: 27$ & 100.0506 \\
$\Delta a=+100 \mathrm{~km}$ & $2020-12-03 \mathrm{~T} 19: 29: 57$ & 99.6085 \\
$e=0.01$ & $2020-12-03 \mathrm{~T} 20: 37: 47$ & 100.0955 \\
$\Delta i=+0.5^{\circ}$ & $2020-12-04 \mathrm{~T} 02: 00: 32$ & 102.5546 \\
\hline
\end{tabular}

响较小; 半长轴增大 $100 \mathrm{~km}$ 影响起飞时间约 $1 \mathrm{~h}$ 、起飞 方位角约 $1^{\circ}$; 倾角增大 $0.5^{\circ}$ 影响起飞时刻约 $5.5 \mathrm{~h}$ 、起 飞方位角约 $2.5^{\circ}$.

\section{7 结论}

本文根据轨道器环月飞行的交会对接轨道和上升
器月面起飞位置, 给出了月面起飞参数初值计算方法. 引入上升器月球交会远程导引控制策略, 给出了基于 轨道共面交会、精确求解月面优化起飞参数的修正算 法和迭代计算流程. 通过仿真算例, 验证了起飞方位角 和起飞时刻初值计算和迭代计算的正确性和快速收敛 性，获得的起飞方位角和起飞时刻有效地消除了远程 导引终点法向偏差, 实现了无需远程导引轨道平面修 正控制量的优化远程导引控制, 节省了上升器推进剂 消耗. 本方法对于起飞点位置和探测器轨道参数变化 具有一定的适用性. 后续将针对探测器在月球极区探 测的起飞开展研究. 本文提出的基于轨道共面交会的 起飞参数计算方法可应用于月球轨道交会对接任务, 以及从其他地外天体起飞实现交会对接的起飞参数确 定, 实现控制量优化的交会控制, 具有较高的工程应用 价值.

\section{参考文献}

1 Ye P J, Guo L L, Zhang Z X, et al. Risks and challenges of manned deep space exploration (in Chinese). Manned Spaceflight, 2016, 22: 143-149 [叶培建, 果琳丽, 张志贤, 等. 有人参与深空探测任务面临的风险和技术挑战. 载人航天, 2016, 22: 143-149]

2 Guo L L, Wang P, Liang L, et al. Preliminary research on manned lunar landing and lifting-off technology (in Chinese). Spacecr Recov Remot Sens, 2013, 34: 10-16 [果琳丽, 王平, 梁鲁, 等. 载人月面着陆及起飞技术初步研究. 航天返回与遥感, 2013, 34: 10-16]

3 Peng J, Huang H, Xiang K H, et al. Conception design of a lunar robotic sampling and return mission (in Chinese). Spacecraft Eng, 2010, 19: 99104 [彭咕, 黄昊, 向开恒, 等. 月球无人采样返回任务概念设想. 航天器工程, 2010, 19: 99-104]

4 Wang W H, Tan T L, He L. Manned spaceflight initial alignment calibration off the lunar surface based on combination navigation (in Chinese). Manned Spaceflight, 2014, 20: 296-300 [王卫华, 谭天乐, 贺亮. 基于组合导航的月面起飞自主对准技术. 载人航天, 2014, 20: 296-300]

5 Betts J T. Survey of numerical methods for trajectory optimization. J Guid Control Dyn, 1998, 21: 193-207

6 Hull D G. Conversion of optimal control problems into parameter optimization problems. J Guid Control Dyn, 1997, 20: 57-60

7 Huang G Q, Lu Y P, Nan Y. A survey of numerical algorithms for trajectory optimization of flight vehicles. Sci China Tech Sci, 2012, 55: 25382560

8 Hull D G. Optimal guidance for quasi-planar lunar ascent. J Optim Theor Appl, 2011, 151: 353-372

9 Hull D G, Harris M W. Optimal solutions for Quasiplanar ascent over a spherical Moon. J Guid Control Dyn, 2012, 35: 1218-1224

10 Li X, Liu Y Y, Zhou J. Design of guidance law for lunar ascent phase of manned lunar module (in Chinese). Syst Eng Electron, 2011, 33: 24802484 [李金金, 刘荣荣, 周军. 载人登月舱上升入轨段的制导律设计. 系统工程与电子技术, 2011, 33: 2480-2484]

11 Ma K M, Chen H P. Optimal trajectory design for the lunar module in ascent stage (in Chinese). Chin Space Sci Tech, 2013, 33: 54-60 [马克茂, 陈海鹏. 登月舱上升段最优轨迹设计. 中国空间科学技术, 2013, 33: 54-60]

12 Zhang L. Trajectory optimization and parameter analysis for lunar ascent (in Chinese). J Deep Space Expl, 2019, 6: 391-397 [张否. 月面上升的 轨迹优化与参数分析. 深空探测学报, 2019, 6: 391-397]

13 Alexander J D, Young K A. Apollo lunar rendezvous. J Spacecr Rockets, 1970, 7: 1083-1086

14 Gutkowski J P, Dawn T F, Jedrey R M. Evolution of Orion mission design for exploration mission 1 and 2. Technical Report. AAS 16-111, JSCCN-35267, 2016

15 Murtazin R. Rendezvous missions: From ISS to lunar space station. Acta Astronaut, 2014, 101: 151-156

16 Chen H, Zhang H L, Han C, et al. Mission planning of fast rendezvous strategy around the Moon (in Chinese). Manned Spaceflight, 2016, 22: 417-422 [陈欢, 张洪礼, 韩潮, 等. 环月远程快速交会任务规划. 载人航天, 2016, 22: 417-422] 
17 Hou Z D, Zhang B N, Tian L, et al. Research on manned lunar ascent rendezvous orbit with relative geometry constraint (in Chinese). Manned Spaceflight, 2019, 25: 481-487 [侯振东, 张柏楠, 田林, 等. 考虑相对几何约束的载人月面上升交会轨道研究. 载人航天, 2019, 25: 481-487]

18 Zhu H, Luo Y Z, Yang Z. Analysis and design of phasing strategy for lunar orbit short rendezvous mission (in Chinese). Manned Spaceflight, 2017, 23: 8-13 [祝海, 罗亚中, 杨震. 环月快速交会调相策略设计与任务分析. 载人航天, 2017, 23: 8-13]

19 Wang Z S, Meng Z F, Gao S. Study of orbit maneuver strategy for lunar orbit rendezvous mission (in Chinese). Spacecr Eng, 2014, 23: 103-110 [汪中生, 孟占峰, 高珊. 月球轨道交会任务的远程导引变轨策略研究. 航天器工程, 2014, 23: 103-110]

20 Chen H P, Yu X H, Huang F. Optimization design of orbit inclination of human lunar landing emergency return trajectory (in Chinese). Chin Space Sci Tech, 2017, 37: 69-74 [陈海朋, 余薛浩, 黄飞. 载人登月应急返回轨道倾角优化设计. 中国空间科学技术, 2017, 37: 69-74]

21 Li G F. Orbit Control Planning for Rendezvous and Docking (in Chinese). Beijing: National Defence Industry Press, 2016 [ 李革非. 空间交会对 接轨道控制规划. 北京: 国防工业出版社, 2016]

\title{
Method for calculation of takeoff parameters of lunar ascent module based on orbital coplanar rendezvous
}

\author{
LI GeFei ${ }^{1,2^{*}} \&$ LIU Yong ${ }^{1,2}$ \\ ${ }^{1}$ Beijing Aerospace Flight Control Center, Beijing 100094, China; \\ 2 Aerospace Flight Dynamics Laboratory, Beijing 100094, China
}

\begin{abstract}
To solve the lunar orbit rendezvous problem, in which the ascent and orbital modules are coplanar to achieve fuel consumption optimization, a method that accurately determines the ascender's takeoff parameters is proposed. Based on the lunar takeoff position of the ascent module and flight orbit of the orbital module, an analytical model for calculating the initial values of the takeoff azimuth and the takeoff time is presented using the spherical triangle relationship of the orbit plane passing through the takeoff site. The injection orbit of the ascent module is calculated based on its lunar ascending characteristic parameters. The four-pulse long-range guidance orbit maneuver strategy is adopted to realize the rendezvous orbit maneuvers of the ascent module. The mapping relationship between the orbital plane deviations of the long-range guidance terminal of the two-spacecraft rendezvous and the orbital parameter deviations of the injection orbit is established. The analytical algorithm for correcting takeoff azimuth and takeoff time based on orbit inclination deviation and the ascending node right ascension deviation is derived. Further, an iterative calculation process for precise lunar takeoff parameters is designed. Simulation results verify the correctness and rapid convergence of the initial value and correction methods for the lunar ascent module's takeoff parameters. The long-range guidance terminal normal deviations are effectively eliminated by the precise lunar takeoff parameters obtained based on the orbital coplanar rendezvous. The optimized rendezvous orbit maneuver without orbital plane correction saves the ascent module's propellant consumption. The method for calculating takeoff parameters can be used to optimize and determine the takeoff parameters for rendezvous and docking from the moon and other extraterrestrial bodies.
\end{abstract}

lunar takeoff, takeoff azimuth, takeoff time, rendezvous and docking, long-range guidance orbit maneuver

PACS: $02.60 . \mathrm{Gf}, 02.60 . \mathrm{Pn}$, 95.55.Pe, $97.75 . \mathrm{Pq}$

doi: $10.1360 /$ SSPMA-2021-0056 